PROCEEDINGS OF THE

AMERICAN MATHEMATICAL SOCIETY

Volume 134, Number 11, November 2006, Pages 3343-3352

S 0002-9939(06)08419-X

Article electronically published on May 12, 2006

\title{
ANALYTICITY FOR SINGULAR SUMS OF SQUARES OF DEGENERATE VECTOR FIELDS
}

\author{
DAVID S. TARTAKOFF
}

(Communicated by Eric Bedford)

Abstract. Recently J. J. Kohn (2005) proved $C^{\infty}$ hypoellipticity for

$$
P_{k}=L \bar{L}+\bar{L}|z|^{2 k} L=-\bar{L}^{*} \bar{L}-\left(\bar{z}^{k} L\right)^{*} \bar{z}^{k} L \quad \text { with } \quad L=\frac{\partial}{\partial z}+i \bar{z} \frac{\partial}{\partial t},
$$

(the negative of) a singular sum of squares of complex vector fields on the complex Heisenberg group, an operator which exhibits a loss of $k-1$ derivatives. Subsequently, M. Derridj and D. S. Tartakoff proved analytic hypoellipticity for this operator using rather different methods going back to earlier methods of Tartakoff. Those methods also provide an alternate proof of the hypoellipticity given by Kohn.

In this paper, we consider the equation

$$
P_{m, k}=L_{m} \overline{L_{m}}+\overline{L_{m}}|z|^{2 k} L_{m} \quad \text { with } L_{m}=\frac{\partial}{\partial z}+i \bar{z}|z|^{2 m} \frac{\partial}{\partial t},
$$

for which the underlying manifold is only of finite type, and prove analytic hypoellipticity using methods of Derridj and Tartakoff. This operator is also subelliptic with large loss of derivatives, but the exact loss plays no role for analytic hypoellipticity. Nonetheless, these methods give a proof of $C^{\infty}$ hypoellipticity with precise loss as well, which is to appear in a forthcoming paper by A. Bove, M. Derridj, J. J. Kohn and the author.

\section{IntroduCtion AND STATEMENT OF THEOREMS}

J.J. Kohn's recent paper [7, inspired by work of Siu 9] on singular metrics and the implied applications, studied a singular sum of squares of complex vector fields on the (complex) Heisenberg group

$$
\begin{gathered}
\left(*_{k}\right) \quad P=-L \bar{L}-\bar{L}|z|^{2 k} L \quad \text { with } \quad L=\frac{\partial}{\partial z}+i \bar{z} \frac{\partial}{\partial t}, \\
\text { i.e., } \\
P=\bar{L}^{*} \bar{L}+\left(\bar{z}^{k} L\right)^{*} \bar{z}^{k} L .
\end{gathered}
$$

He showed that this operator was hypoelliptic but loses $k-1$ derivatives (in Sobolev norms), and a note [3] by Christ showed that, as in the Baouendi-Goulaouic example, hypoellipticity is destroyed by adding an elliptic contribution in a new variable.

Subsequently, in [5], M. Derridj and D. S. Tartakoff proved analytic hypoellipticity for this operator using rather different methods going back to [10, 11]. These methods provide an alternate proof of the main result in [7] (cf. [1]).

Received by the editors June 1, 2005.

2000 Mathematics Subject Classification. Primary 35H10; Secondary 35N15.

(C)2006 American Mathematical Society Reverts to public domain 28 years from publication 
In this paper, we consider the more degenerate equation

$\left(*_{m, k}\right) \quad P=P_{m}=L_{m} \overline{L_{m}}+\overline{L_{m}}|z|^{2 k} L_{m} \quad$ with $L_{m}=\frac{\partial}{\partial z}+i \bar{z}|z|^{2 m} \frac{\partial}{\partial t}$,

for which the underlying manifold is only of finite type, and prove analytic hypoellipticity for this operator.

Theorem 1. The operator $P_{m}$ is analytic hypoelliptic: $P_{m} u \in C^{\omega} \rightarrow u \in C^{\omega}$.

Remark 1. We note that in a forthcoming paper, together with A. Bove, M. Derridj and J. J. Kohn, we will utilize these methods to give a proof of the $C^{\infty}$ hypoellipticity for these operators.

\section{The a priori ESTIMATE}

As in [5] we work with $v \in C_{0}^{\infty}$ and denote by $\Lambda$ the 'pseudodifferential' operator with symbol $\lambda(z, t ; \zeta, \tau)=\left(1+|\tau|^{2}\right)^{1 / 2}$. Since $\Lambda$ acts in the $t$ variable alone, negative powers of $\Lambda$ do not have negative order in the usual Sobolev spaces. However, from the point of view of analytic hypoellipticity this will not matter, since for $\zeta$ different from zero the operator $P_{m}$ is elliptic, hence analytic hypoelliptic, near $z=0$, and for $z$ different from 0 , the operator $P_{m}$ has symplectic characteristic variety and satisfies maximal estimates in $L$ and $\bar{L}$, hence is even analytic hypoelliptic there by [10, [12, 11]. An important property of $\Lambda$ is that it commutes with $P_{m}$, since the latter is independent of $t$.

Thus we will need only study the behavior of powers of $\partial / \partial t$ applied to the solution $u$ locally. We shall do so in $L^{2}$ norms with appropriate powers of $\Lambda$.

The a priori estimate satisfied by $P_{m}$ is actually better than that satisfied by $P_{0}$, but as is well known, analyticity does not use the precise degree of subellipticity in an important way.

We begin by giving a short proof of the main estimate of [7].

Proposition 1. For all values of $m$, we have

$$
\left\|\overline{L_{m}} v\right\|^{2}+\left\|\bar{z}^{k} L v\right\|^{2}+\|v\|_{-\frac{k-1}{2}}^{2} \leq C\left|\left(P_{m} v, v\right)\right|, \quad v \in C_{0}^{\infty} .
$$

For any $r<0$, since $\Lambda$ commutes with everything, we have

$$
\left\|\Lambda^{r} v\right\|^{2}=\left(\left[L_{m}, z\right] \Lambda^{r} v, \Lambda^{r} v\right)=\left(z \Lambda^{r} v, \Lambda^{r} \overline{L_{m}} v\right)-\left(L_{m} \Lambda^{r-\frac{1}{2}} v, \bar{z} \Lambda^{r+\frac{1}{2}} v\right),
$$

SO

$$
\left\|\Lambda^{r} v\right\|^{2} \leq \text { s.c. }\left\|\Lambda^{r} v\right\|^{2}+\text { l.c. }\left\|\overline{L_{m}} v\right\|^{2}+\text { s.c. }\left\|L_{m} \Lambda^{r-\frac{1}{2}} v\right\|^{2}+\text { l.c. }\left\|\bar{z} \Lambda^{r+\frac{1}{2}} v\right\|^{2} .
$$

Now integrating by parts and commuting $L_{m}$ and $\overline{L_{m}}$ we have

$$
\left\|L_{m} \Lambda^{r-\frac{1}{2}} v\right\|^{2} \leq\left\|\overline{L_{m}} \Lambda^{r-\frac{1}{2}} v\right\|^{2}+\left\|\Lambda^{r} v\right\|^{2},
$$

so that (2.1) becomes

$$
\left\|\Lambda^{r} v\right\|^{2} \lesssim\left\|\overline{L_{m}} v\right\|^{2}+\left\|\bar{z} \Lambda^{r+\frac{1}{2}} v\right\|^{2} \lesssim\left|\left(P_{m} v, v\right)\right|+\left\|\bar{z} \Lambda^{r+\frac{1}{2}} v\right\|^{2} .
$$

In the last term we integrate by parts, and obtain

$$
\begin{gathered}
\left\|\bar{z} \Lambda^{r+\frac{1}{2}} v\right\|^{2}=\left(\bar{z} \Lambda^{r+\frac{1}{2}} v, \bar{z} \Lambda^{r+\frac{1}{2}} v\right)=\left(z \bar{z} \Lambda^{r+1} v, \Lambda^{r} v\right) \\
\leq \text { s.c. }\left\|\Lambda^{r} v\right\|^{2}+\text { l.c. }\left\|z^{2} \Lambda^{r+1} v\right\|^{2}
\end{gathered}
$$

so that, iterating and absorbing the first term on the left in (2.2),

$$
\left\|\Lambda^{r} v\right\|^{2} \lesssim\left|\left(P_{m} v, v\right)\right|+\left\|z^{k-1} \Lambda^{r+\frac{k-1}{2}} v\right\|^{2} .
$$


To finish the derivation of the a priori estimate, we write $k z^{k-1}=\left[L_{m}, z^{k}\right]$, so that

$$
\begin{aligned}
k^{2}\left\|z^{k-1} w\right\|^{2} & =\left(\left[L_{m}, z^{k}\right] w, z^{k-1} w\right) \\
=\left(L_{m} z^{k} w, z^{k-1} w\right) & -\left(z^{k} L_{m} w, z^{k-1} w\right)=\tilde{A}+\tilde{B} .
\end{aligned}
$$

Then

$$
|\tilde{A}| \lesssim\left|\left(z^{k-1} w, \bar{z} \overline{L_{m}} z^{k-1} w\right)\right| \lesssim s . c .\left\|z^{k-1} w\right\|^{2}+\text { l.c. }\left\|\overline{L_{m}} w\right\|^{2}
$$

since $\left[\overline{L_{m}}, z^{k-1}\right]=0$, while

$$
|\tilde{B}| \sim \text { s.c. }\left\|z^{k-1} w\right\|^{2}+\text { l.c. }\left\|z^{k} L_{m} w\right\|^{2} .
$$

The terms with the small constant (s.c.) will be absorbed on the left-hand side of (2.4), yielding

$$
\left\|z^{k-1} w\right\|^{2} \leq C\left\{\left\|\overline{L_{m}} w\right\|^{2}+\left\|\bar{z}^{k} L_{m} w\right\|^{2}\right\}
$$

Combining this estimate with (2.1), since $\left\|z^{k} w\right\|=\left\|\bar{z}^{k} w\right\|$, leads to

$$
\left\|\Lambda^{-\frac{k-1}{2}} v\right\|^{2} \leq C\left\{\left\|\overline{L_{m}} v\right\|^{2}+\left\|\bar{z}^{k} L_{m} v\right\|^{2}\right\}=C\left|\left(P_{m} v, v\right)\right| .
$$

Remark 1. If one wishes to use the full pseudodifferential operator with symbol $\left(1+|\zeta|^{2}+|\tau|^{2}\right)^{1 / 2}$ instead of $\Lambda$, the derivation is virtually identical, with the addition of a lower order norm on the right of (2.6).

\section{The localization of powers of $T$ And its Commutators}

To apply (2.6) we must replace $v$ by a localization of powers of $\partial / \partial t$, or, for convenience, we will localize powers of

$$
T=-\frac{2 i}{m+1} \frac{\partial}{\partial t}
$$

applied to the solution $u$. Note that

$$
\left[L_{m}, \overline{L_{m}}\right]=(m+1)^{2}|z|^{2 m} T,
$$

which will be of technical use later.

For now we treat the solution $u$ as being smooth, and concentrate on the estimates, remarking that by multiplying the Fourier Transform $\tilde{u}(z, \bar{z}, \tau)$ of $u$ in $t$ by dilations of $\Psi(\tau)$ equal to zero for $|\tau|>2$ but identically equal to one for $|\tau|<1$, once we obtain uniform estimates (in $\varepsilon$ ) for derivatives of the inverse transform of $\Psi(\tau / \varepsilon)(\tilde{u})$ we may let $\varepsilon \rightarrow 0$ to obtain the same estimates for the same derivatives of $u$.

The localization in space may be taken independent of $z, \bar{z}$, since taking a product of two localizers, $\varphi_{1}(t) \varphi_{2}(z, \bar{z})$, in terms where no derivatives land on $\varphi_{2}$, it just survives to the left of $P_{m}=f$, while in terms with derivatives, those terms are supported in the region where $z$ is different from zero, i.e., in regions where the result is known, as remarked above.

But even with $\varphi$ depending on $t$ alone, the localization must be done very carefully. For example, the first bracket with $P_{m}$ which we encounter will contain $\left(L_{m} \varphi(t)\right) T^{p} \sim \bar{z}|z|^{2 m} \varphi^{\prime} T^{p}$, which is problematic for any value of $m$.

In Kohn's work 7] $(m=0)$, the $\bar{z}$ (or $z)$ in front of each derivative of $\varphi$ is carefully followed, and shown to provide, after some work, a gain of $1 / 2$ derivative. Analyticity was not considered in that paper, nor does it seem likely that it could be shown by those methods. 
Derridj and Tartakoff found in [5] that an entirely different approach, involving a delicately balanced localization of $T^{p}$, led to analyticity rather directly.

Here, to handle the case $m>0$, which corresponds to a CR manifold of finite type, we find it simpler to use a somewhat differently balanced localization. With this localization both $C^{\omega}$ and $C^{\infty}$ hypoellipticity come together.

3.1. The case $m=0$. For motivation, and to show how much simpler the example is in the contact case, we first give the definition in the case $m=0$.

Definition 3.1. For any pair of non-negative integers, $\left(p_{1}, p_{2}\right)$, let

$$
\left(T_{0}^{p_{1}, p_{2}}\right)_{\varphi}=\sum_{\substack{a \leq p_{1} \\ b \leq p_{2}}} \frac{{\overline{L_{m}}}^{a} \circ \bar{z}^{a} \circ T^{p_{1}-a} \circ \varphi^{(a+b)} \circ T^{p_{2}-b} \circ z^{b} \circ L_{m}^{b}}{a ! b !},
$$

where

$$
\varphi^{(r)}=\left(-i \frac{\partial}{\partial t}\right)^{r} \varphi .
$$

Note that the leading term (with $a+b=0$ ) is merely $T^{p_{1}} \circ \varphi \circ T^{p_{2}}$, which is equal to the operator $T^{p_{1}+p_{2}}$ on any open set $\Omega_{0}$ where $\varphi \equiv 1$.

We have the precise commutation relations:

\section{Proposition 3.1.}

$$
\begin{aligned}
{\left[L_{0},\left(T_{0}^{p_{1}, p_{2}}\right)_{\varphi}\right] } & \equiv\left(T_{0}^{p_{1}, p_{2}-1}\right)_{\varphi^{\prime}} \circ L_{0}, \\
{\left[L_{0},\left(T_{0}^{p_{1}, p_{2}}\right)_{\varphi}\right] } & \equiv \overline{L_{0}} \circ\left(T_{0}^{p_{1}-1, p_{2}}\right)_{\varphi^{\prime}}, \\
{\left[\left(T_{0}^{p_{1}, p_{2}}\right)_{\varphi}, z\right] } & =z \circ\left(T_{0}^{p_{1}, p_{2}-1}\right)_{\varphi^{\prime}},
\end{aligned}
$$

and

$$
\left[\left(T_{0}^{p_{1}, p_{2}}\right)_{\varphi}, \bar{z}\right]=\left(T_{0}^{p_{1}-1, p_{2}}\right)_{\varphi^{\prime}} \circ \bar{z},
$$

where the $\equiv$ denotes modulo $C^{p_{1}-p_{1}^{\prime}+p_{2}-p_{2}^{\prime}}$ terms of the form

$$
\frac{{\overline{L_{0}}}^{p_{1}-p_{1}^{\prime}} \circ \bar{z}^{p_{1}-p_{1}^{\prime}} \circ T^{p_{1}^{\prime}} \circ \varphi^{\left(p_{1}^{\prime}+p_{2}^{\prime}+1\right)} \circ T^{p_{2}^{\prime}} \circ z^{p_{2}-p_{2}^{\prime}} \circ L_{0}{ }^{p_{2}-p_{2}^{\prime}}}{\left(p_{1}-p_{1}^{\prime}\right) !\left(p_{2}-p_{2}^{\prime}\right) !}
$$

with either $p_{1}^{\prime}=0$ or $p_{2}^{\prime}=0$, i.e., terms where all free $T$ derivatives have been eliminated on one side of $\varphi$ or the other.

Proof. The proof is a straightforward calculation involving a shift of index in the definition of $\left(T_{0}^{p_{1}, p_{2}}\right)_{\varphi}$.

3.2. The case $m>0$. For $m>0$, we use a related, but somewhat different definition of the localization, owing to multiple brackets. Parts of this discussion are in the style of [4, [2].

Definition 3.2. For $m>0$, and $\left(p_{1}, p_{2}\right)$ as above, set

$$
\begin{gathered}
\varphi^{(d)}=\left(i \frac{\partial}{\partial t}\right)^{d} \varphi(t), \\
N_{b}=\sum_{b^{\prime} \leq b} A_{b^{\prime}}^{b} \frac{\left(z L_{m}\right)^{b^{\prime}}}{b^{\prime} !},
\end{gathered}
$$


where the $A_{b^{\prime}}^{b}$ (real) are to be determined subject to $A_{b}^{b}=1$,

$$
\tilde{N}_{a}=\sum_{a^{\prime} \leq a} A_{a^{\prime}}^{a} \frac{\left(-\overline{L_{m}} \bar{z}\right)^{a^{\prime}}}{a^{\prime} !}=\left(N_{a}\right)^{*}
$$

with the same coefficients, and finally we set

$$
\left(T_{m}^{p_{1}, p_{2}}\right)_{\varphi}=\sum_{\substack{a \leq p_{1} \\ b \leq p_{2}}} \tilde{N}_{a} \circ T^{p_{1}-a} \circ \varphi^{(a+b)} \circ T^{p_{2}-b} \circ N_{b} .
$$

We have

$$
\begin{gathered}
{\left[\overline{L_{m}},\left(T_{m}^{p_{1}, p_{2}}\right)_{\varphi}\right]=\left[\overline{L_{m}}, \sum_{a \leq p_{1}, b \leq p_{2}} \tilde{N}_{a} T^{p_{1}-a} \varphi^{(a+b)} T^{p_{2}-b} N_{b}\right]} \\
=\sum_{a \leq p_{1}, b \leq p_{2}}\left\{\left[\overline{L_{m}}, \tilde{N}_{a}\right] T^{p_{1}-a} \varphi^{(a+b)} T^{p_{2}-b} N_{b}\right. \\
\left.-\tilde{N}_{a} T^{p_{1}-a} z|z|^{2 m} \varphi^{(a+b+1)} T^{p_{2}-b} N_{b}+\tilde{N}_{a} T^{p_{1}-a} \varphi^{(a+b)} T^{p_{2}-b}\left[\overline{L_{m}}, N_{b}\right]\right\} .
\end{gathered}
$$

The last two terms on the right must cancel, to preserve the balance, since both disturb the balance between derivatives on $\varphi$ and gain in powers of $T$. We will choose the coefficients $A_{b^{\prime}}^{b}$ of $N_{b}$ in such a way that, modulo acceptable errors,

$$
\left[\overline{L_{m}}, N_{b}\right]=z|z|^{2 m} T N_{b-1} \text {. }
$$

This will provide the needed cancellation via a shift of index in $a$ in the sum just as in the case with $m=0$. The corresponding relation for brackets with $L_{m}$ will follow by taking adjoints: again modulo acceptable errors,

$$
\left[L_{m}, \tilde{N}_{a}\right]=-\tilde{N}_{a-1} \bar{z}|z|^{2 m} T .
$$

Condition (3.2), using the definition of $N_{b}$, reads:

$$
\sum_{b^{\prime}=0}^{b} A_{b^{\prime}}^{b} \frac{1}{b^{\prime} !}\left[\overline{L_{m}},\left(z L_{m}\right)^{b^{\prime}}\right]=-\bar{z}|z|^{2 m} T \sum_{b^{\prime}=0}^{b-1} A_{b^{\prime}}^{b-1} \frac{\left(z L_{m}\right)^{b^{\prime}}}{b^{\prime} !} .
$$

Expanding the brackets and keeping all factors of $z L_{m}$ to the right,

$$
\begin{gathered}
\frac{1}{b^{\prime} !}\left[\overline{L_{m}},\left(z L_{m}\right)^{b^{\prime}}\right]=\frac{1}{b^{\prime} !} \sum_{1 \leq b^{\prime \prime} \leq b^{\prime}}\left(\begin{array}{c}
b^{\prime} \\
b^{\prime \prime}
\end{array}\right) a d_{z L_{m}}^{b^{\prime \prime}}\left(\overline{L_{m}}\right)\left(z L_{m}\right)^{b^{\prime}-b^{\prime \prime}} \\
=\frac{1}{b^{\prime} !} \sum_{1 \leq b^{\prime \prime} \leq b^{\prime}}\left(\begin{array}{c}
b^{\prime} \\
b^{\prime \prime}
\end{array}\right) a d_{z L_{m}}^{b^{\prime \prime}-1}\left((m+1)^{2} z|z|^{2 m} T\right)\left(z L_{m}\right)^{b^{\prime}-b^{\prime \prime}} \\
=\left(z|z|^{2 m} T\right) \sum_{1 \leq b^{\prime \prime} \leq b^{\prime}} \frac{(m+1)^{b^{\prime \prime}+1}}{b^{\prime \prime} !} \frac{\left(z L_{m}\right)^{b^{\prime}-b^{\prime \prime}}}{\left(b^{\prime}-b^{\prime \prime}\right) !}
\end{gathered}
$$

The condition (3.2) thus requires, renaming $b^{\prime}-b^{\prime \prime}$ as $\bar{b}$ on the right just above,

$$
\sum_{b^{\prime \prime}=1}^{b-\tilde{b}} A_{\tilde{b}+b^{\prime \prime}}^{b} \frac{(m+1)^{b^{\prime \prime}+1}}{b^{\prime \prime} !}=A_{\tilde{b}}^{b-1} .
$$

Fortunately, we have investigated these equations in [4] and, citing a result in the book by Hirzebruch [6], have explicit solutions $\tilde{A}_{*}^{*}$ in the case that $m=0$, unique 
under the conditions that $\tilde{A}_{0}^{q}=(-1)^{q}$, namely

$$
\tilde{A}_{s}^{r}=\left(\left(\frac{t}{e^{t}-1}\right)^{r+1}\right)^{(r-s)}(0) /(r-s) !
$$

and for positive $m$ we merely dilate by the factor of $m+1$ :

$$
A_{s}^{r}=\left(\left(\frac{\frac{t}{m+1}}{e^{\frac{t}{m+1}}-1}\right)^{r+1}\right)^{(r-s)}(0) /(r-s) !
$$

In addition, we will also need good expressions for the other brackets: we compute

$$
\begin{gathered}
{\left[L_{m}, N_{b}\right]=\left[L_{m}, \sum_{b^{\prime}=0}^{b} A_{b^{\prime}}^{b} \frac{\left(z L_{m}\right)^{b^{\prime}}}{b^{\prime} !}\right]=\sum_{\substack{1 \leq b^{\prime \prime} \leq b^{\prime} \\
b^{\prime} \leq b}} A_{b^{\prime}}^{b} \frac{1}{b^{\prime \prime \prime} !} \frac{(z L)^{b^{\prime}-b^{\prime \prime}}}{\left(b^{\prime}-b^{\prime \prime}\right) !} \circ L_{m},} \\
{\left[N_{b}, z\right]=z \circ \sum_{\substack{1 \leq b^{\prime \prime} \leq b^{\prime} \\
b^{\prime} \leq b}} A_{b^{\prime}}^{b} \frac{1}{b^{\prime \prime} !} \frac{(z L)^{b^{\prime}-b^{\prime \prime}}}{\left(b^{\prime}-b^{\prime \prime}\right) !},} \\
{\left[\overline{L_{m}}, \tilde{N}_{a}\right]=-\overline{L_{m}} \circ \sum_{\substack{1 \leq a^{\prime \prime} \leq a^{\prime} \\
a^{\prime} \leq a}} A_{a^{\prime}}^{a} \frac{1}{a^{\prime \prime \prime} !} \frac{\left(-\overline{L_{m}} \bar{z}\right)^{a^{\prime}-a^{\prime \prime}}}{\left(a^{\prime}-a^{\prime \prime}\right) !},} \\
{\left[\bar{z}, \tilde{N}_{a}\right]=\sum_{\substack{1 \leq a^{\prime \prime} \leq a^{\prime} \\
a^{\prime} \leq a}} A_{a^{\prime}}^{a} \frac{1}{a^{\prime \prime !} !} \frac{\left(-\overline{L_{m}} \bar{z}\right)^{a^{\prime}-a^{\prime \prime}}}{\left(a^{\prime}-a^{\prime \prime}\right) !} \circ \bar{z} .}
\end{gathered}
$$

In order to recognize these sums as $N$ 's or $\tilde{N}$ 's, we need to be able to shift the lower indices on $A_{a^{\prime}}^{a}$ down by one. But we have also done this in [4, with the result that

Proposition 3.2. For any $r, s$, and $c$,

$$
A_{s}^{r}=\sum_{j=0}^{r-s} S_{j}^{r-s} A_{s-c}^{r-(c+j)}
$$

where

$$
\left|S_{\ell}^{k}\right| \leq C^{k}
$$

These brackets, then, together with the proposition, immediately translate, setting $b^{\prime \prime}=c$ and $\tilde{b}=b^{\prime}-b^{\prime \prime}$, into

$$
\left[L_{m}, N_{b}\right]=\sum_{\tilde{b} \leq b-c} \frac{1}{c !} A_{\tilde{b}+c}^{b} \frac{(z L)^{\tilde{b}}}{\tilde{b} !} \circ L_{m}=\sum_{\tilde{b} \leq b-c-j} \frac{1}{c !} S_{j}^{c+j} A_{\tilde{b}}^{b-c-j} \frac{(z L)^{\tilde{b}}}{\tilde{b} !} \circ L_{m}
$$

or

$$
\left[L_{m}, N_{b}\right]=\sum_{\substack{c+j \leq b \\ 1 \leq c}} \frac{1}{c !} S_{j}^{c+j} N_{b-c-j} \circ L_{m}
$$


Similarly,

$$
\begin{gathered}
{\left[\overline{L_{m}}, \tilde{N}_{a}\right]=-\overline{L_{m}} \circ \sum_{\substack{c+j \leq a \\
1 \leq c}} \frac{1}{c !} S_{j}^{c+j} \tilde{N}_{a-c-j},} \\
{\left[\bar{z}, \tilde{N}_{a}\right]=\sum_{\substack{c+j \leq a \\
1 \leq c}} \frac{1}{c !} S_{j}^{c+j} \tilde{N}_{a-c-j} \circ \bar{z},}
\end{gathered}
$$

and

$$
\left[N_{b}, z\right]=z \circ \sum_{\substack{c+j \leq b \\ 1 \leq c}} \frac{1}{c !} S_{j}^{c+j} \tilde{N}_{b-c-j}
$$

These precise commutation relations mean that the whole localization $\left(T^{p_{1}, p_{2}}\right)_{\varphi}$ may be commuted meaningfully with the vector fields $L_{m}, \overline{L_{m}}$ and with $z, \bar{z}$.

Proposition 3.3. Modulo terms in which either $p_{1}$ or $p_{2}$ has been reduced to zero, and in view of the cancellations ensured by (3.2),

$$
\left[L_{m},\left(T_{m}^{p_{1}, p_{2}}\right)_{\varphi}\right] \equiv \sum_{\substack{1 \leq c, 0 \leq j \\ c+j \leq p_{2}}} \frac{1}{c !} S_{j}^{c+j}\left(T_{m}^{p_{1}, p_{2}-(c+j)}\right)_{\varphi(c+j)} \circ L_{m} .
$$

Proof.

$$
\begin{gathered}
{\left[L_{m},\left(T_{m}^{p_{1}, p_{2}}\right)_{\varphi}\right] \equiv \sum_{\substack{a \leq p_{1} \\
b \leq p_{2}}} \tilde{N}_{a} \circ T^{p_{1}-a} \circ \varphi^{(a+b)} \circ T^{p_{2}-b}\left[L_{m}, N_{b}\right]} \\
\equiv \sum_{\substack{a \leq p_{1} \\
b \leq p_{2}}} \sum_{\substack{c+j \leq b \\
1 \leq c}} \frac{1}{c !} S_{j}^{c+j} \tilde{N}_{a} \circ T^{p_{1}-a} \circ \varphi^{(a+b)} \circ T^{p_{2}-b} N_{b-c-j} \circ L_{m} \\
\equiv \sum_{\substack{a \leq p_{1} \\
b \leq p_{2}}} \sum_{\substack{c+j \leq b \\
1 \leq c}} \frac{1}{c !} S_{j}^{c+j} \tilde{N}_{a} T^{p_{1}-a} \varphi^{(c+j)^{(a+b-c-j)}} T^{p_{2}-(c+j)-(b-c-j)} N_{b-c-j} \circ L_{m} \\
\equiv \sum_{\substack{1 \leq c, 0 \leq j \\
c+j \leq p_{2}}} \frac{1}{c !} S_{j}^{c+j}\left(T_{m}^{p_{1}, p_{2}-(c+j)}\right)_{\varphi^{(c+j)}} \circ L_{m} .
\end{gathered}
$$

Similarly we state the following proposition, and omit the proofs, which are virtually identical to that of the previous proposition.

\section{Proposition 3.4.}

$$
\begin{aligned}
{\left[\overline{L_{m}},\left(T_{m}^{p_{1}, p_{2}}\right)_{\varphi}\right] } & \equiv-\overline{L_{m}} \circ \sum_{\substack{1 \leq c, 0 \leq j \\
c+j \leq p_{1}}} \frac{1}{c !} S_{j}^{c+j}\left(T_{m}^{p_{1}-(c+j), p_{2}}\right)_{\varphi^{(c+j)}}, \\
{\left[\bar{z},\left(T_{m}^{p_{1}, p_{2}}\right)_{\varphi}\right] } & \equiv \sum_{\substack{1 \leq c, 0 \leq j \\
c+j \leq p_{1}}} \frac{1}{c !} S_{j}^{c+j}\left(T_{m}^{p_{1}-(c+j), p_{1}}\right)_{\varphi^{(c+j)}} \circ \bar{z},
\end{aligned}
$$

and

$$
\left[z,\left(T_{m}^{p_{1}, p_{2}}\right)_{\varphi}\right] \equiv-\sum_{\substack{1 \leq c, 0 \leq j \\ c+j \leq p_{2}}} \frac{1}{c !} S_{j}^{c+j}\left(T_{m}^{p_{1}, p_{2}-(c+j)}\right)_{\varphi^{(c+j)}} \circ z .
$$


What these commutation relations mean is that we may move the vector fields of $P_{m}$ past $\left(T_{m}^{p_{1}, p_{2}}\right)_{\varphi}$ freely, at each stage incurring errors with the same vector fields and a gain in derivatives in $\left(T_{m}^{p_{1}, p_{2}}\right)_{\varphi}$. Thus we may iterate the a priori inequality modulo errors of nearly arbitrarily low order - all of the $\equiv$ signs above mean that we will ultimately arrive at errors where either $p_{1}=0$ or $p_{2}=0$.

So we first insert $v=\left(T_{m}^{p_{1}, p_{2}}\right)_{\varphi} u$ into (2.6), then bring $\left(T_{m}^{p_{1}, p_{2}}\right)_{\varphi}$ to the left of $P_{m}=L \bar{L}+\bar{L} z^{k} \bar{z}^{k} L$, now writing $L$ instead of $L_{m}$, since the formal expansions of the brackets are insensitive to $m$. Therefore, we have

$$
\begin{gathered}
\left\|\bar{L}\left(T_{m}^{p_{1}, p_{2}}\right)_{\varphi} u\right\|_{0}^{2}+\left\|\bar{z}^{k} L\left(T_{m}^{p_{1}, p_{2}}\right)_{\varphi} u\right\|_{0}^{2}+\left\|\Lambda^{-\frac{k-1}{2}}\left(T_{m}^{\frac{p}{2}, \frac{p}{2}}\right)_{\varphi} u\right\|_{0}^{2} \\
\lesssim\left|\left(P_{m}\left(T_{m}^{p_{1}, p_{2}}\right)_{\varphi} u,\left(T_{m}^{p_{1}, p_{2}}\right)_{\varphi} u\right)_{L^{2}}\right| \\
\lesssim\left|\left(\left(T_{m}^{p_{1}, p_{2}}\right)_{\varphi} P_{m} u,\left(T_{m}^{p_{1}, p_{2}}\right)_{\varphi} u\right)_{L^{2}}\right|+\left|\left(\left[P_{m},\left(T_{m}^{p_{1}, p_{2}}\right)_{\varphi}\right] u,\left(T_{m}^{p_{1}, p_{2}}\right)_{\varphi} u\right)_{L^{2}}\right|,
\end{gathered}
$$

and by the above bracket relations, modulo the same terms as above where all $T$ 's from one side of $\varphi$ or the other have been 'converted' into $L$ 's or $\bar{L}$ 's, we have

$$
\begin{gathered}
\left(\left[P_{m},\left(T_{m}^{p_{1}, p_{2}}\right)_{\varphi}\right] u,\left(T_{m}^{p_{1}, p_{2}}\right)_{\varphi} u\right) \\
=\left(\left[L \bar{L},\left(T^{p_{1}, p_{2}}\right)_{\varphi}\right] u,\left(T^{p_{1}, p_{2}}\right)_{\varphi} u\right)+\left(\left[\bar{L} z^{k} \bar{z}^{k} L,\left(T^{p_{1}, p_{2}}\right)_{\varphi}\right] u,\left(T^{p_{1}, p_{2}}\right)_{\varphi} u\right) \\
=\left(\left[L,\left(T^{p_{1}, p_{2}}\right)_{\varphi}\right] \bar{L} u,\left(T^{p_{1}, p_{2}}\right)_{\varphi} u\right)+\left(L\left[\bar{L},\left(T^{p_{1}, p_{2}}\right)_{\varphi}\right] u,\left(T^{p_{1}, p_{2}}\right)_{\varphi} u\right) \\
+\left(\left[\bar{L},\left(T^{p_{1}, p_{2}}\right)_{\varphi}\right] z^{k} \bar{z}^{k} L u,\left(T^{p_{1}, p_{2}}\right)_{\varphi} u\right)+\left(\bar{L}\left[z^{k},\left(T^{p_{1}, p_{2}}\right)_{\varphi}\right] \bar{z}^{k} L u,\left(T^{p_{1}, p_{2}}\right)_{\varphi} u\right) \\
+\left(\bar{L} z^{k}\left[\bar{z}^{k},\left(T^{p_{1}, p_{2}}\right)_{\varphi}\right] L u,\left(T^{p_{1}, p_{2}}\right)_{\varphi} u\right)+\left(\bar{L} z^{k} \bar{z}^{k}\left[L,\left(T^{p_{1}, p_{2}}\right)_{\varphi}\right] u,\left(T^{p_{1}, p_{2}}\right)_{\varphi} u\right) \\
\equiv \sum_{\substack{1 \leq c, 0 \leq j \\
c+j \leq p_{2}}} \frac{1}{c !} S_{j}^{c+j}\left(\left(T_{m}^{p_{1}, p_{2}-(c+j)}\right)_{\varphi}^{(c+j)} L \bar{L} u,\left(T_{m}^{p_{1}, p_{2}}\right)_{\varphi} u\right) \\
-\sum_{\substack{1 \leq c, 0 \leq j \\
c+j \leq p_{1}}} \frac{1}{c !} S_{j}^{c+j}\left(L \bar{L}\left(T_{m}^{p_{1}-(c+j), p_{2}}\right)_{\varphi}^{(c+j)} u,\left(T_{m}^{p_{1}, p_{2}}\right)_{\varphi} u\right) \\
-\sum_{\substack{1 \leq c, 0 \leq j \\
c+j \leq p_{1}}} \frac{1}{c !} S_{j}^{c+j}\left(\bar{L}\left(T_{m}^{p_{1}-(c+j), p_{2}}\right)_{\varphi^{(c+j)}} z^{k} \bar{z}^{k} L u,\left(T_{m}^{p_{1}, p_{2}}\right)_{\varphi} u\right) \\
+\sum_{k^{\prime}=1}^{k} \sum_{\substack{1 \leq c, 0 \leq j \\
c+j \leq p_{2}}} \frac{1}{c !} S_{j}^{c+j}\left(\bar{L} z^{k^{\prime}}\left(T_{m}^{p_{1}, p_{2}-(c+j)}\right)_{\varphi(c+j)} z^{k-k^{\prime}} \bar{z}^{k} L u,\left(T_{m}^{p_{1}, p_{2}}\right)_{\varphi} u\right) \\
+\sum_{k^{\prime}=0}^{k-1} \sum_{\substack{1 \leq c, 0 \leq j \\
c+j \leq p_{1}}} \frac{1}{c !} S_{j}^{c+j}\left(\bar{L} z^{k} \bar{z}^{k^{\prime}}\left(T_{m}^{p_{1}-(c+j), p_{1}}\right)_{\varphi(c+j)} \bar{z}^{k-k^{\prime}} L u,\left(T_{m}^{p_{1}, p_{2}}\right)_{\varphi} u\right) \\
+\sum_{\substack{1 \leq c, 0 \leq j \\
c+j \leq p_{2}}} \frac{1}{c !} S_{j}^{c+j}\left(\bar{L} z^{k} \bar{z}^{k}\left(T_{m}^{p_{1}, p_{2}-(c+j)}\right)_{\varphi(c+j)} L u,\left(T_{m}^{p_{1}, p_{2}}\right)_{\varphi} u\right) \\
=A_{1}+A_{2}+A_{3}+\sum_{k^{\prime}=1}^{k} A_{4, k^{\prime}}+\sum_{k^{\prime}=0}^{k-1} A_{5, k^{\prime}}+A_{6} .
\end{gathered}
$$

Concerning the critical $L, \bar{L}, z^{k}$ and $\bar{z}^{k}$, note that in each term above

- No $L, \bar{L}$, power of $z$ or power of $\bar{z}$ has been lost.

- The order among $L, \bar{z}^{k}, z^{k}$, and $\bar{L}$ is preserved. 
- Letting $|q|=q_{1}+q_{2}$, each term on the right contains $\left(T^{q_{1}, q_{2}}\right)_{\varphi(|p|-|q|)}$ with $|q|<|p|$ and $|p|-|q|$ derivatives on $\varphi$.

- Just as (3.11) demonstrates the errors which result in moving $\left(T^{p_{1}, p_{2}}\right)_{\varphi}$ past the vector fields $L, \overline{L_{m}}, z^{k} \overline{L_{m}}$ and $\bar{z}^{k} L$, further such brackets used to position the vector fields so as to make use of the a priori estimate again will produce similar errors, with $|q|$ still lower and the 'lost' $T$ derivatives transferred to $\varphi$.

- Iterating this process, together with a weighted Schwarz inequality, will produce a sum of terms with $q_{j} \leq \frac{p}{2}$ of the form

$$
\text { l.c. } \left.\left\|\Lambda^{\frac{k-1}{2}}\left(T_{m}^{q_{1}, q_{2}}\right)_{\varphi(|p|-|q|)} P_{m} u\right\|_{0}^{2}+\text { s.c. } \| \Lambda^{-\frac{k-1}{2}}\left(T_{m}^{\frac{p}{2}, \frac{p}{2}}\right)_{\varphi} u\right) \|^{2} .
$$

- In fact, using larger constants $\tilde{S}_{j}^{c+j}$ subject to the same kind of bounds, $\left|\tilde{S}_{j}^{c+j}\right| \leq \tilde{C}^{c+j}$, we may replace all sums on the right-hand side above by suprema subject to the same range restrictions on the indices.

- This use of suprema allows us to easily iterate everything on the right with easy control on the constants until either $p_{1}$ or $p_{2}$, both of which start as $\frac{p}{2}$, drops to zero, which may happen in two ways - either by stepwise decrease as on the right-hand side above from successive brackets, or by the single term in Propositions 3.1 and 3.3 which is not cancelled, the term with all $L$ 's or $\bar{L}$ 's on one side or the other in the definition of $\left(T_{m}^{p_{1}, p_{2}}\right)_{\varphi}$, whose principal term is $\left(T_{m}^{p_{1}, 0}\right)_{\varphi^{\left(p_{2}\right)}}(z L)^{p_{2}} / p_{2}$ ! or its analogue with $p_{1}$ is reduced to 0 .

- At this point we no longer have an effective localization of powers of $T$ - for example, brackets with $\bar{L}$ are not corrected. We proceed anyway, and when we lack a 'good' vector field such as $\bar{L}$ (or of course $\bar{z}^{k} L$ ), we create one by integrating by parts

$$
\|L w\|^{2} \leq\|\bar{L} w\|^{2}+\left|\left(|z|^{2 m} T w, w\right)\right|
$$

to use up the $L$ and $\bar{L}$ derivatives with the byproduct of introducing up to half the number of new $T$ derivatives.

Overall, then, the strategy has been, with universal $C$, bounding derivatives of the Ehrenpreis-type localizing functions $\varphi^{(r)}$ by $(C N)^{r} \sim C^{r} r$ ! for $r \leq N \sim p$ :

$$
\begin{gathered}
\frac{\left\|\Lambda^{-\frac{k-1}{2}} T^{p} u\right\|_{\{\varphi \equiv 1\}}}{N^{p}} \rightarrow \frac{\left\|\Lambda^{-\frac{k-1}{2}}\left(T_{m}^{\frac{p}{2}, \frac{p}{2}}\right)_{\varphi} u\right\|}{N^{p}} \\
\rightarrow C^{\frac{p}{4}} \frac{\left\|\Lambda^{-\frac{k-1}{2}} T^{\frac{3 p}{4}} u\right\|_{s u p p \varphi}}{N^{\frac{3 p}{4}}} \rightarrow C^{\frac{p}{4}} \frac{\left\|\Lambda^{-\frac{k-1}{2}} T^{\frac{3 p}{4}} u\right\|_{\left\{\varphi_{1} \equiv 1\right\}}}{N^{\frac{3 p}{4}}} \rightarrow \ldots
\end{gathered}
$$

for suitable $\varphi_{1} \equiv 1$ on the support of $\varphi$. This will continue, with a sequence of $\varphi_{j}$ supported in nested intervals as in [10, 11] until only a negligible fraction of $p$ is left, namely a bounded number of derivatives. Since the order in $T^{p}$ is reduced by a factor of $3 / 4$ each time, we will need $\log _{4 / 3} p$ such nested open sets. Thus (where the constant $C_{P_{m} u}$ reflects the analytic growth of derivatives of $P_{m} u$ ):

$$
\frac{\left\|\Lambda^{-\frac{k-1}{2}} T^{p} u\right\|_{\{\varphi \equiv 1\}}}{N^{p}} \lesssim C^{p}\left\|\Lambda^{-\frac{k-1}{2}+3} u\right\|_{\left\{\varphi_{\log _{4 / 3} p} \equiv 1\right\}}+C_{P_{m} u}^{p}
$$


where the 3 could be any other small integer. Of course the whole derivation could have been done at the $H^{s}$ level: for any given $s$,

$$
\frac{\left\|\Lambda^{s-\frac{k-1}{2}} T^{p} u\right\|_{\{\varphi \equiv 1\}}}{N^{p}} \lesssim C^{p}\left\|\Lambda^{s-\frac{k-1}{2}+3} u\right\|_{\left\{\varphi_{\log _{4 / 3} p} \equiv 1\right\}}+C_{P_{m} u}^{p},
$$

which will end the story if this last norm is known to be finite even as the localizer in $\tau$ tends to the identity, provided that the terms that arise along the way are all similarly bounded. The most important of these is of course

$$
\left|\left(\Lambda^{s+\frac{k-1}{2}}\left(T_{m}^{\frac{p}{2}, \frac{p}{2}}\right)_{\varphi} P u, \Lambda^{s-\frac{k-1}{2}}\left(T_{m}^{\frac{p}{2}, \frac{p}{2}}\right)_{\varphi} u\right)\right|,
$$

which shows that $P_{m} u \in H^{s+\frac{k-1}{2}+p}$ implies that $u \in H^{s-\frac{k-1}{2}+p}$, a loss of $k-1$ derivatives.

Remark 1. The value of $s$ will be chosen so that we know the norm on the right in (3.15) is finite as the localizer in $\tau$ goes to the identity, and then $p$ will be chosen so that $P_{m} u \in H^{s+\frac{k-1}{2}+p}$ for that value of $s$. It follows that $u \in H^{s-\frac{k-1}{2}+p}$.

Remark 2. For analyticity, one needs to ensure that as we take $p$ larger and larger, the constants satisfied by the Ehrenpreis-type localizers are subject to bounds such that the estimate (3.15) is uniform in $p$. We have shown this often before (cf. 10], [11]), and the arguments are the same here.

\section{REFERENCES}

[1] A. Bove, M. Derridj, J.J. Kohn and D.S. Tartakoff, Hypoellipticity for a sum of squares of complex vector fields with large loss of derivatives, preprint.

[2] A. Bove And D.S. TARTAKoff, Analytic hypo-ellipticity at non-symplectic characteristics when the symplectic form changes its rank, preprint.

[3] M Christ, A remark on sums of squares of complex vector fields, preprint, arXiv:math.CV/0503506.

[4] M. DerRidj AND D.S. TARTAKofF, Local analyticity for $\square_{b}$ and the $\bar{\partial}$-Neumann problem at certain weakly pseudoconvex points, Commun. in Partial Differential Equations 13(12) (1988), pp. 1521-1600. MR0970155 (89m:35158)

[5] M. Derridu and D.S. Tartakoff, Analyticity and loss of derivatives, Annals of Mathematics 162(2) (2005), as Appendix to Hypoellipticity and loss of derivatives, by J. J. Kohn in the same issue.

[6] F. Hirzebruch, Topological Methods in Algebraic Geometry, Springer-Verlag, New York, 1966. MR0202713 (34:2573)

[7] J.J. Konn, Hypoellipticity and loss of derivatives, Annals of Mathematics 162(2) (2005).

[8] C. Parenti and A. Parmeggiani, A Note on Kohn's and Christ's Examples, preprint.

[9] Y.-T. Siu, Extension of twisted pluricanonical sections with plurisubharmonic weight and invariance of semipositively twisted plurigenera for manifolds not necessarily of general type, in Complex Geometry: Collection of Papers Dedicated to Professor Hans Grauert, 223-277, Springer-Verlag, New York, 2002. MR1922108 (2003j:32027a)

[10] D.S. TARTAKoff, Local analytic hypoellipticity for $\square_{b}$ on non-degenerate Cauchy Riemann manifolds, Proc. Nat. Acad. Sci. U.S.A. 75 (1978), pp. 3027-3028. MR0499657(80g:58045)

[11] D.S. TARTAKOFF, On the local real analyticity of solutions to $\square_{b}$ and the $\partial$-Neumann problem, Acta Math. 145 (1980), pp. 117-204. MR0590289 (81k:35033)

[12] F. Treves, Analytic hypo-ellipticity of a class of pseudo-differential operators with double characteristics and application to the $\bar{\partial}$-Neumann problem, Comm. in P.D.E. 3 (6-7) (1978), pp. 475-642. MR0492802 (58:11867)

Department of Mathematics, University of Illinois at Chicago, m/C 249, 851 S. Morgan Street, Chicago, Illinois 60607

E-mail address: dst@uic.edu 